\title{
Special Polymers for Data Memories
}

\author{
G. KAEMPF \\ Bayer AG, Department of Technical Development/Applied Physics, \\ D-5090 Leverkusen (FRG)
}

(Received August 18, 1986)

\begin{abstract}
Microelectronics represent to a large extent the key industry in the $80^{\prime}$ and $90^{\prime} \mathrm{s}$ of this century. Progress in microelectronics, however, is directly related to progress in data storage technology. Up to now main data memories are exclusively based on silicon semiconductor devices, mass data memories - at least the most important ones- are magnetic data storage units. New on the market are non-erasable optical memories using polymers or polymer substrates (e.g., CDROM's). Under development are erasable optical and electrical polymer data memories: photopolymers, LC-side chain polymers, ferroelectric polymers, and such for holographic or photochemical hole burning data storage. The paper describes the current state of art and compares the various developments of special polymers in an attempt to forecast the possible significance of polymer memories with high data packing densities in relation to the established technologies.

KEY WORDS Data Memories / Data Storage / Polymers / Dyes / Substrate Materials /
\end{abstract}

Technological developments in the 1990's will be determined by progress in the field of microelectronics. Microelectronics has become the motivating force of innovation, the deciding factor in competitiveness and the key to new products. ${ }^{1}$

One of the essential microelectronic assemblies is the data storage unit of memory. Data memories are the heart of every modern computer. There are on the one hand main memories for moderate data volumes and extremely short access time, on the other hand mass memories for very large data volumes and relatively short access time. Today, main memories are almost exclusively silicon semiconductor memories. The most important memory type are read only memories (ROM) and $\mathrm{read} / \mathrm{write}$ memories (RAM: random access memory). Figure 1 illustrates the economic significance of semiconductor memories which account for more than half of the total microelectronics market for integrated circuits. As can be seen, in 1998 the market volume for semiconductor memories worldwide will account for $53 \%$ of US $\$ 232$ billion, i.e. approx. US\$120 billion. That is roughly half of the current automobile market. ${ }^{1}$

The most important mass memories are magnetic storage units with data packing densities of approx. $10^{6} \mathrm{bit} / \mathrm{cm}^{2}$, for instance, magnetic tapes and magnetic disks (floppy or hard disk).

In 1985, the optical storage disk (CD-ROM) was introduced to the market. Its technology is identical with the audio compact disk (CD): the PCM signals (pulse code modulated signals) are embossed directly into the polymer substrate. This is done during the injection molding process by using a special mold insert (stamper), which transfers the microscopic small pits into the disk surface. The information is read out using a low energy laser, which reads through the transparent disk. The sequence of reflection and non-reflection, caused by the pit sequence in the disk surface is used as binary code. A CD-ROM with a disk 


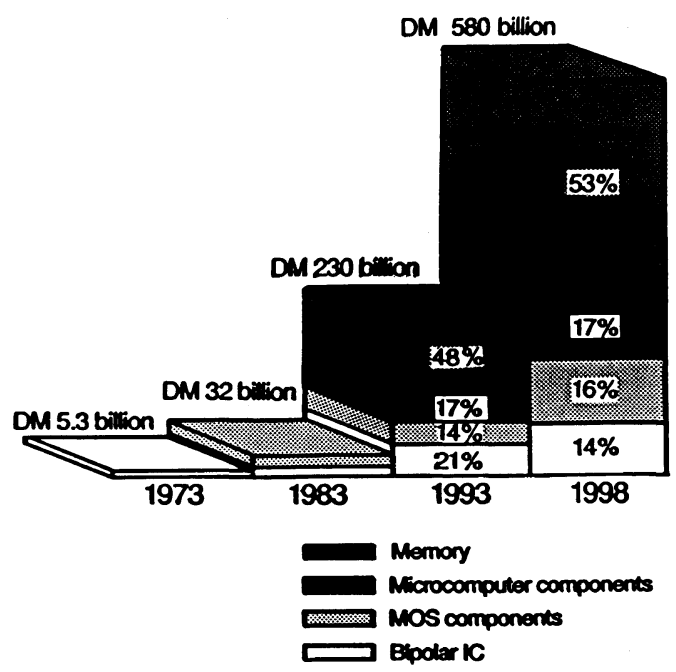

Figure 1. Economic significance of the semiconductor memory, illustrated by the development of the world market for integrated circuits.

diameter of $4.75^{\prime \prime}$ has a storage capacity of 550 Mbyte, a disk with $12^{\prime \prime}$ in diameter stores up to 3 GByte which is equivalent to 630,000 typewritten pages. ${ }^{2}$

\section{Non Erasable Optical Data Memories Using \\ Polymers or Polymer Substrates}

Current developments of non erasable media are going in different directions. ${ }^{2}$ :

a) Pits embossed into polymer material during manufacturing process. Example: CDROM.

b) Pits burned into polymer materials, containing light-absorbing additives (organic dyestuffs, organic and inorganic pigments, carbon black).

c) Pits burned into thin metal layers on transparent substrates (glass or plastics). Example: TeSe (Philips DOR system $=$ Digital Optical Recording).

d) Production of bubbles in thin metal layers on transparent substrates (glass or plastics). Examples: Te alloys (Hitachi, Bell, 3M, OSI, Optimen Div.); Au/Pt alloy (Thompson-CSF).

e) Indentations fused into thin layers of organic dyestuffs in polymer binders on transparent substrates: Examples: Squarylium-derivates e.g., SQS (Philips), ${ }^{3}$ Carbocyanine (Eastman-Kodak).

The technologies, described under a), b), and e) are total polymer data memories, c) and d) describe systems that use only polymer substrates.

\section{Erasable Optical Data Memories Using Poly- mer Substrates}

Interesting attempts are being made to overcome the disadvantages of present day optomechanical memories, namely the high energies required for writing and, in particular, their lack of erasability and reuse. Which of the technologies described below will finally be successful, depends on the requirements imposed for each of them. The most important criteria, against which all developments are measured, are $^{2}$ :

- Extended life time ( $>15$ years for mass memories, particulary in the archival and documentation area).

- High read-erase-write speed $\left(>10^{6} \mathrm{bit} / \mathrm{s}\right)$.

- Low material fatigue, great number of write/erase cycles ( $>10^{7}$ cycles).

- Low cost per bit and low hardware costs.

- Low fault susceptibility ( $10^{7}$ bit/error without necessary error correction, $10^{12}$ bit/error with error correction).

- Easy handling.

- Good market acceptance, e.g., compatibility with existing data processing systems.

Erasable optical storage systems using polymer substrates currently under development today are:

- Magneto Optical memories (MO systems): thin amorphous layers with reversible magnetization. Examples: FeGdCo (Philips); GDTbFe (Matsushita, Philips, Xerox), TbDyFe (Sharp), TbFeCo (Hitachi, Sony); FeTb (IBM).

-Phase-Change memories (PC systems): reversible amorphous-crystalline phase transitions of metal or metal oxide layers. Exam- 


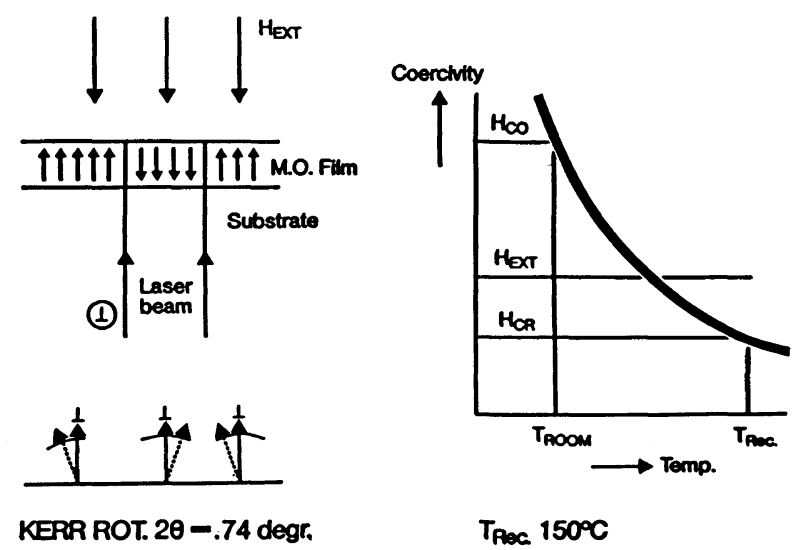

Figure 2. Magneto-optical memory (MO-system): Magnetic reversal process and signal reading procedure.

ples: Te-alloys (Philips), $\mathrm{TeTeO}_{2}$ (Matsushita). - Reversible color and/or reflection changes thermally induced by lasers. Examples: AgZn alloys (Hitachi), phtalocyanin derivatives in PE or PST (IBM, TDK), photochromic substances in polymer matrix (Bell).

Of these types, the magneto-optical system has been developed furthest; the first trial samples are already being launched on the market (Optimen Div., Storage Corp., $3 \mathrm{M}$ ).

Figure 2 shows a diagrammatic sketch of the structure of a magneto-optical disk, its magnetic reversal process and its signal reading procedure: a suitable transparent, almost birefringence-free polymer substrate (PC, PMMA, etc.) is coated with an approx. 20 $\mathrm{nm}$ thick amorphous magnetic layer (composed of $\mathrm{FeGdCo}$, for example). The data are written by heating the layer briefly to a temperature close to or above the Curie point with the aid of a laser beam focused to approx. $1 \mu \mathrm{m}^{2}$, so that the binary bit signal can be written by an external magnetic field as a correspondingly assigned magnetization direction. The data are read out optically by means of a finely-focused polarized laser beam; depending on magnetization direction, the rotation of the plane of polarization will differ (Kerr effect), this being detected optically. The disadvantage of the MO sys-

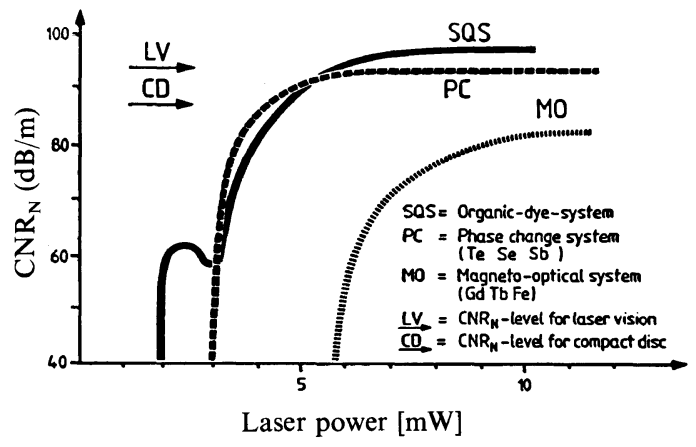

Figure 3. Carrier-to-noise ratio $\left(\mathrm{CNR}_{\mathrm{N}}\right)$ on read out of different erasable optical data storage system as a function of the writing energy (laser power). (By courtesy of Philips Research Laboratories, Eindhoven, The Netherlands.)

tem is the very small angle of rotation of the plane of polarization during reading, this resulting in an unsatisfactory signal-to-noise ratio $\mathrm{CNR}_{\mathrm{N}}$ (Figure 3$){ }^{4}$

In the phase-change system, the data storage disk again consists of a suitable transparent, low-birefringence polymer substrate (PC, PMMA, etc.), to which a thin crystalline metal or metal-oxide layer is applied (e.g., Te-Se$\mathrm{Sb}$ or Te suboxide). The data are written by briefly melting the crystalline layer by means of a finely-focused laser beam, this resulting in a local phase transition from crystalline to 

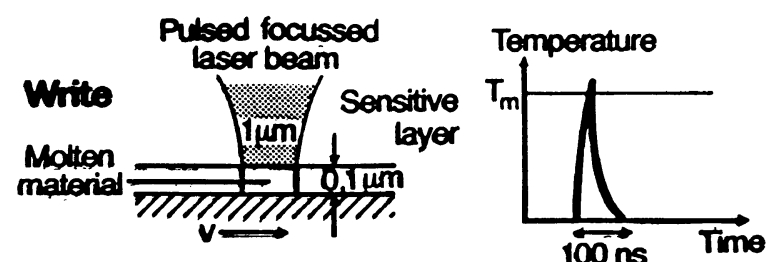

Top viow
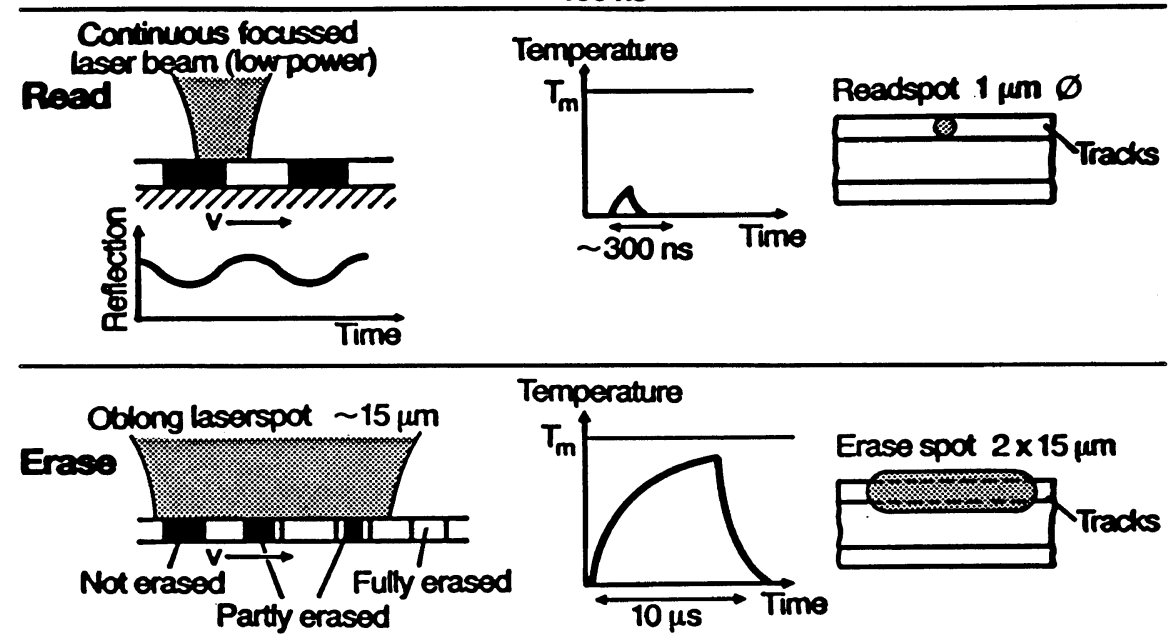

Figure 4. Phase-change optical memory write-/read-/erase-cycle (Philips, The Netherlands).

amorphous (Figure 4). ${ }^{4}$ The stored data are read out optically, utilizing the different light-scattering behaviour of the crystalline and amorphous regions. Difficulties are encountered in erasing the data, i.e., in reversing the phase from amorphous to crystalline by brief annealing. The laser beam is enlarged accordingly in order to guarantee the required annealing time (from $1 \mu \mathrm{m}^{2}$ to $18 \times 2 \mu \mathrm{m}^{2}$, refer to Figure 4$)$. The signal-to-noise ratio of the PC-system is slightly above the $\mathrm{CNR}_{\mathrm{N}}$ of the MO system (Figure 3 ). ${ }^{4}$

Polymer layers containing dyestuffs using photochromic substances may become extremely important as erasable data memories in the future. ${ }^{23}$ Examples include optically switchable bistable, organic dyes, such as fulgides (developed by Prof. Heller, Univ. of Bristol, Great Britain), like those shown in Figure $5,,^{5}$ or metal complex pigments (e.g., nickel metal complex cationic pigment, developed by TDK, Japan), or colour centres in



Figure 5. Absorption spectra of a bistable photochromic dye (Fulgide) in bleached state (erased) and colored state (written); after Heller.

alkali halide crystals. ${ }^{6}$ Dye-polymer-systems are of special interest: the active memory layer can be applied by familiar spin coating techniques, this resulting in low production costs. 


\begin{tabular}{|c|c|c|c|c|c|}
\hline Properties & glass & PMMA & $\mathrm{PC}$ & PS & PVC \\
\hline Heat resistance & 0 & $\Delta$ & 0 & $\Delta$ & 미 \\
\hline $\begin{array}{l}\text { Absorption of water } \\
\text { (warping) }\end{array}$ & 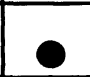 & E & $\Delta$ & 0 & O \\
\hline $\begin{array}{l}\text { Sensivity against } \\
\text { birefringence }\end{array}$ & 0 & 0 & $\square$ & - & $\Delta$ \\
\hline $\begin{array}{l}\text { Light transmiltance } \\
\text { (optical quality) }\end{array}$ & 0 & 0 & $\Delta$ & 0 & $\Delta$ \\
\hline Density (meigth) & E & $\mathrm{O}$ & $\mathrm{O}$ & 0 & $\Delta$ \\
\hline $\begin{array}{l}\text { Mechanical strength } \\
\text { (toughness) }\end{array}$ & - & $\Delta$ & 0 & & O \\
\hline Scratch resistance & 0 & $\mathrm{O}$ & $\square$ & $\Delta$ & $\Delta$ \\
\hline chemical solvent resistance & $\mathrm{O}$ & $\Delta$ & $\Delta$ & - & $\Delta$ \\
\hline Processing & - & $\mathrm{O}$ & $\Delta$ & $\mathrm{O}$ & $\Delta /$ \\
\hline cost & $\overline{-}$ & $\mathrm{O}$ & $\Delta$ & $\mathrm{O}$ & $\mathrm{O}$ \\
\hline 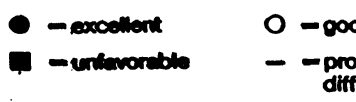 & ons & & & & \\
\hline
\end{tabular}

Figure 6. Qualitative comparison of different substrate materials for optical memory disks (after Hennig).

Furthermore, non-erasable optical data memories based on SQS are known to have a better signal-to-noise ratio than PC and MO systems (Figure 3). However, many problems are still unsolved, such as the stability of the dyes, and the need for the development of diode lasers working in the required visible range ( $\geq 360 \mathrm{~nm}$ ).

Up to now, none of the afore mentioned technologies for erasable optical memories are widely accepted in the market, although the developments are progressing rapidly.

Requirements on Special Polymers as Substrate Materials for Optical Memories

Stringent requirements are imposed on the polymer material for CD-ROM's and for non erasable respectively erasable optical memories $^{2,7}$ :

- High optical homogeneity (low birefringence) and maximum purity.

- High transmission in the visible range and possibly also in the UV range between 280 and $380 \mathrm{~nm}$ ( $2 \mathrm{P}$ process for manufacturing video discs).



Figure 7. Birefringence of a CD-disk (mean value) (measurements perpendicular and horizontal (2 orthogonal directions) related to the disk plane).

- Maximum dimensional stability (minimum thermal expansion, water absorption $<0.3 \%$ ). -Isotropic expansion when exposed to temperature and moisture; excessive water absorption causes warping.

- Mechanical rigidity, high impact strength and toughness.

- High dimensional stability when exposed to heat. 


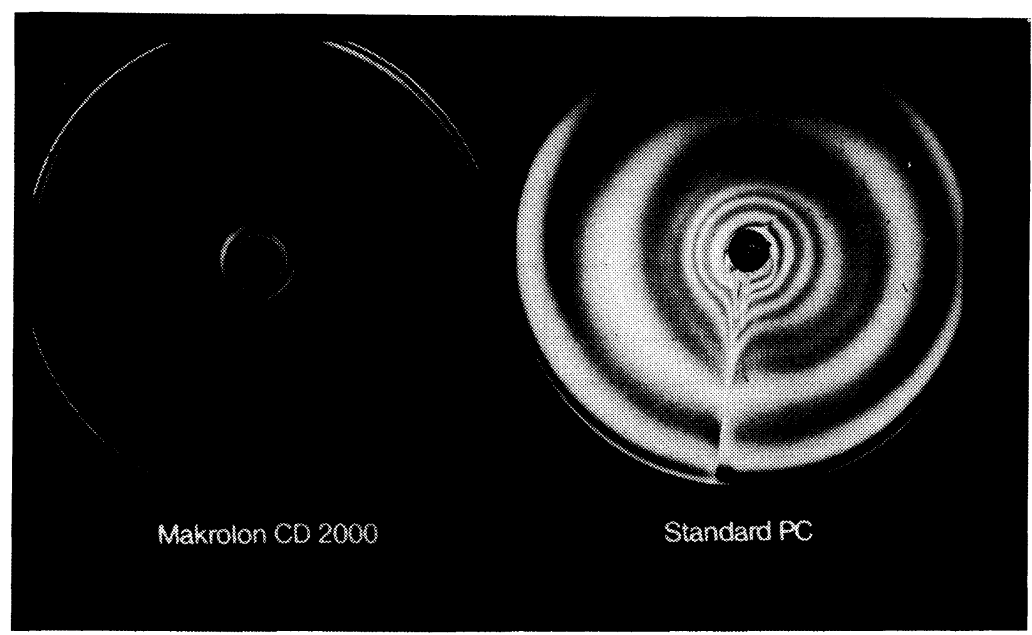

Figure 8. Distribution of stress in a compact disc (photographs taken with circular polarized light).

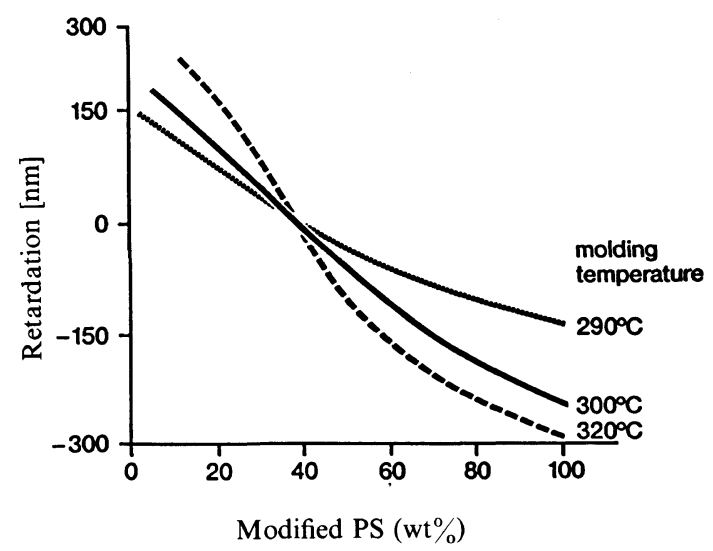

Figure 9. Effect of blend ratio on birefringence of a PC/modif. PS blend (after Sumitomo Chem. Co., Ltd.).

- Very tight thickness tolerances as-moulded disk, surface smoothness.

- Specifically for CD-ROM's: high and constant flow characteristics of the resin to ensure exact pit replication $(0.12$ micron depth, 0.4 0.6 micron width, $0.5-1.5$ micron length).

At the moment, polycarbonate and polymethylmethacrylate are generally used as substrates for ROM optical disks, although the use of PVC, polystyrene, CAB (cellulose acetobutyrate), BDPA (bisdiallyl-polycarbonate), PETP., PMP (poly-4-methyl-pentene)



Figure 10. Birefringence $\Delta n$ of unaxial stretched $\left(l / l_{0}=2\right)$ styrene-butadiene-copolymer as a function of compound composition.

and SAN is also being considered. ${ }^{2}$ The use of modified epoxy is a new possibility offering advantages in thermal stability but disadvantages in economic manufacturing. 9 Figure 6 lists the major advantages and disadvantages of selected polymer materials (PC, PMMA, PS, PVC) in comparison with glass. $^{7}$

Up to now, all CD-ROM's are made from special polycarbonate, as this material offers the best property balance to meet the standards and material specifications. ${ }^{10}$

The benefit of PMMA having low sensitivity to orientation birefringence is opposed by the disadvantage of high water absorption 
causing warp. It is just the inverse situation with PC. ${ }^{8}$

The major disadvantage of $\mathrm{PC}$ is its high intrinsic birefringence. The development of modified polycarbonate grades, e.g., Markolon CD 2000 from Bayer (FRG) or from Mobay Corp. (USA), ${ }^{10}$ and the introduction of modified manufacturing techniques for CD disks with low molecule orientation (so-called injection stamping technique) have allowed successful mass production of almost birefringence-free PC disks $(\Delta n \leqq 20 \mathrm{~nm})$ (cf. Figures 7 and 8).

Another way of obtaining birefringencefree polymer substrates is to blend transparent polymers with positive birefringence (e.g., PC, PVC, PETP, PBD, PVDF) with polymers having negative birefringence (e.g., PMMA, PS, PAN), as in the PC/modified PS blend from Sumitomo, Japan, ${ }^{25}$ (Figure 9) and the PVC/PMMA blend of Roehm, FRG, or to manufacture suitable copolymers (e.g., styrene-butadiene copolymer: Figure 10). ${ }^{8,11}$

A blend of PMMA and PVDF is particularly interesting, since, if blended at an appropriate ratio, it is not only birefringencefree (Figure 11), ${ }^{8,12}$ but also displays considerably reduced water absorption in comparison with pure PMMA.

On optical data disks recorded on one side only, excessive water absorption results in unacceptable warping. Copolymers made of MMA/CHMA (=methylmethacrylate and cyclohexylmethacrylate) or MMA/S (=methyl-



Figure 11. Birefringence $\Delta n$ of unaxial stretched $\left(l / l_{0}=2\right)$ samples of PMMA/PVDF-blends as a function of compound composition. methacrylate with styrene) display a considerable reduction in water absorption, but the material strength is remarkably lowered (MMA/CMMA) or the birefringence is increased (MMA/S). ${ }^{8,25}$

\section{Polymers for Possible Developments in Eras-} able Polymer Data Memories

The following possible developments in polymer data memories are currently being debated $^{2}$ :

- Memories with 2-dimensional data storage.

- amorphous thermoplastics

- crystallizable polymers

- photopolymers

- liquid-crystalline polymers

- ferroelectric polymers

- Memories with 3-dimensional data storage.

- volume holograms in polymers

- photochemical "holeburning" in polymers

The following is a brief outline of the most interesting developments at the moment.

a) Erasable Memories Based on Polymers

With Liquid Crystalline Side Chains

The liquid-crystalline state is a transition between the three-dimensional structure of a crystal and the structure of an isotropic liquid characterized only by short-range orders. In their mesomorphous phases, liquidcrystalline molecules display structural order and molecular mobility. The structural order in certain temperature and concentration ranges results from spontanous reorganisation of the molecules.

The various states of order and the orientation capabilities of liquid-crystalline materials can be utilized for reversible data storage. One simple method of presenting information optically is to generate optical scattering centers in an optically clear liquid crystalline layer. ${ }^{13}$

Liquid crystalline side chain polymers containing dyestuffs covalently linked with the polymer chain are particulary suitable for storage purposes (Figure 12). ${ }^{14}$ Figure 13 il- 


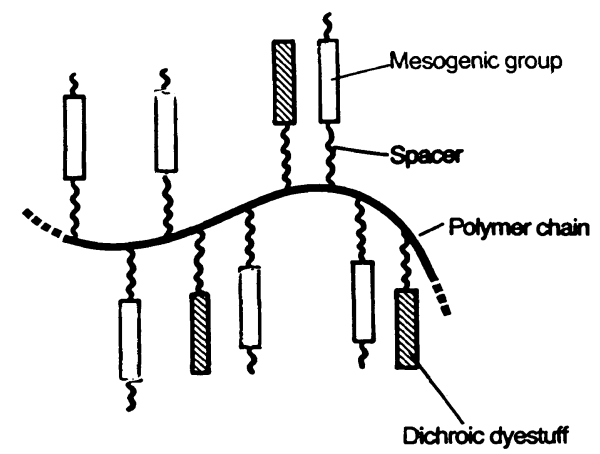

Figure 12. Schematic structure of liquid crystalline side-group polymers containing dyestuff.

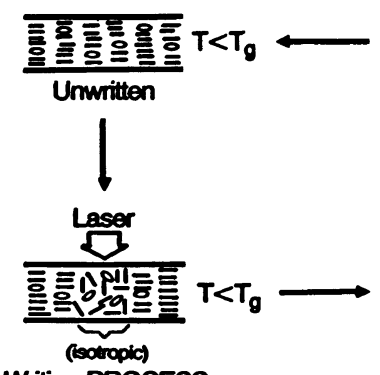

Writing PROCESS

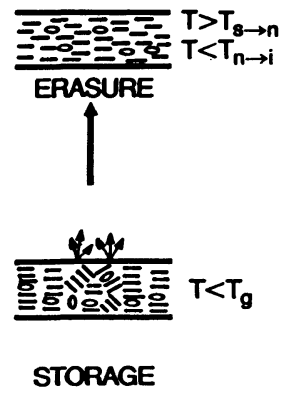

Figure 13. Data memory based on a liquid crystalline side-group polymer containing dyestuff: write/store/ erase cycle.

lustrates the individual stages involved in the writing and erasure process. Initial trials have obtained track width of $10 \mu \mathrm{m}$ in polymer layers of $8 \mu \mathrm{m}$ thickness using a $4 \mathrm{~mW}$ laser, focussed to $5 \mu \mathrm{m}$.

b) Erasable Memories Based on Ferroelectric Polymers

Ferrimagnetic and ferromagnetic storage units (tapes, disks, drums) are used almost exclusively as the mass memories of current computer systems. Analogies between the behavior of ferromagnetic materials in a magnetic field and that of ferroelectric materials in an electric fiẹld suggest that ferroelectric materials could conceivably be used for storage purposes. The relationship between the electric polarisation $P$ and the electric field strength $E$ of ferroelectric materials is described by a hysteresis curve (Figure 14). The

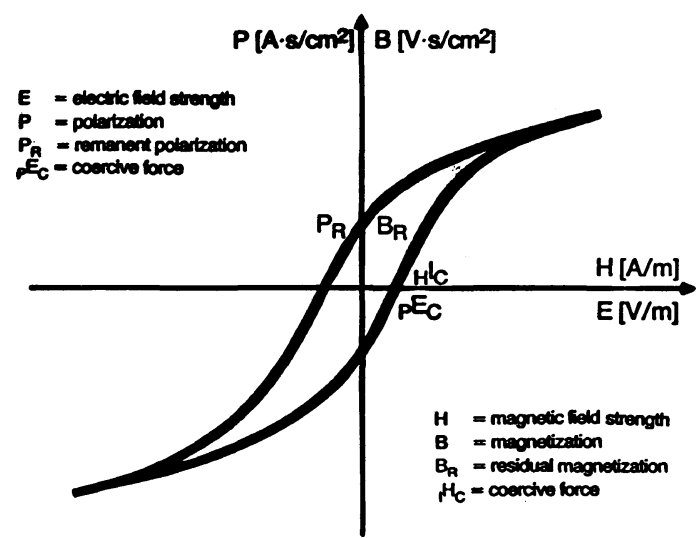

Figure 14. Typical hysteresis curve of a ferromagnetic or ferroelectric material.
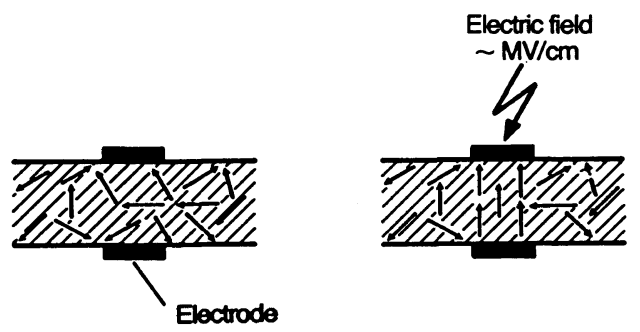

Figure 15. Ferroelectric polymer: permanent local polarization by alignment of the electric dipole in the electric field.

remanent polarisation $P_{\mathrm{R}}$ and the coercive force $\mathrm{pEc}$ are characteristic parameters of the ferroelectrical material. The reversibility of remanent polarisation makes ferroelectric materials suitable for use as analog or binary storage media.

In the early 1970 s, it was found that certain polymers possess suitable piezoelectric and pyroelectric properties; ferroelectric properties were also demonstrated in some polymers. ${ }^{15}$ This is true for polymers whose monomers have large dipole moments and are structured in such a way that they eliminate internal compensation of the dipole moments. Today the most extensively researched polymers with excellent ferroelectric properties are PVDF and PVDF copolymers, the beta-phase of PVDF having a remanent polarization $P_{\mathrm{R}}$ of $5 \mathrm{nC} \mathrm{cm}^{-2}$ and a coercitive force of $\mathrm{pEc}$ of 5 
$\mathrm{MV} / \mathrm{cm}$.

In ferroelectric materials, the writing process (that is the polarization of domains) is accomplished by applying a strong electric field of the order of $\mathrm{MV} \mathrm{cm}{ }^{-1}$ (Figure 15).

For reading, a distinction must be made between reading processes that destroy the information and processes which preserve it. In destructive reading, the originally written information is read out by determining the polarisation current to be applied. ${ }^{16}$ In nondestructive reading either the piezoelectric effect or the pyroelectric effect is used, which means an electric charge corresponding to the permanent polarisation is liberated by applying heat or presure to the storage medium. This charge can be detected by appropriate methods.

The following read and write processes have been described in the literature (Figure 16). ${ }^{2}$

- Reading and writing using electrically conductive electrodes.

- Reading and writing using a metal tip. ${ }^{18}$

- Reading and writing using a laser beam. ${ }^{19}$

-Reading and writing using an electron beam $^{20}$

It is reasonable to expect that ferroelectric polymers will have a role as information sto-

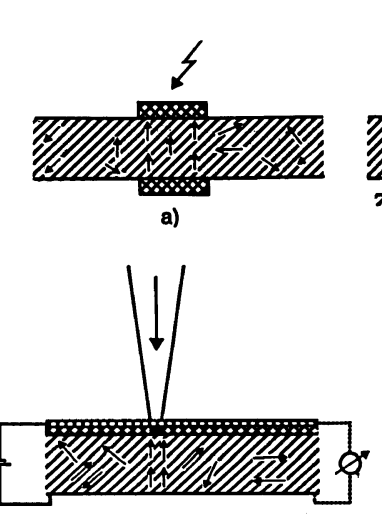

c)

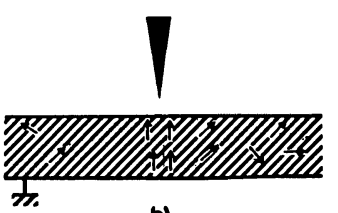

b)

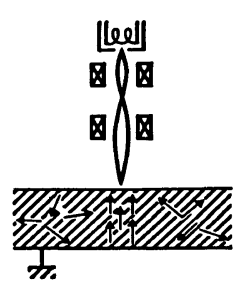

d)
Figure 16. Ferroelectric polymer data memory. Local polarization by means of a) metal electrodes, b) metal tip, c) laser beam, and d) electron beam. rage units in the future; however, a great deal of basic research and development work is still necessary.

c) Erasable Memories Based on Photochemical "Hole-Burning" in Polymers

Another form of three-dimensional information storage developed in recent years by IBM and further developed by Prof. Haarer (Univ. of Bayreuth, FRG) is that of storing data according to area and frequency by the "hole-burning" method. ${ }^{21}$

Absorption experiments on amorphous polymers, such as suitable transparent polymers (e.g., PMMA) containing dyestuff molecules have revealed very inhomogenously broadened spectral lines. This broadening is caused by the energetic interaction of the dyestuff molecules with their highly diverse local environments in the polymer matrix. The ratio of the homogenous (original) line

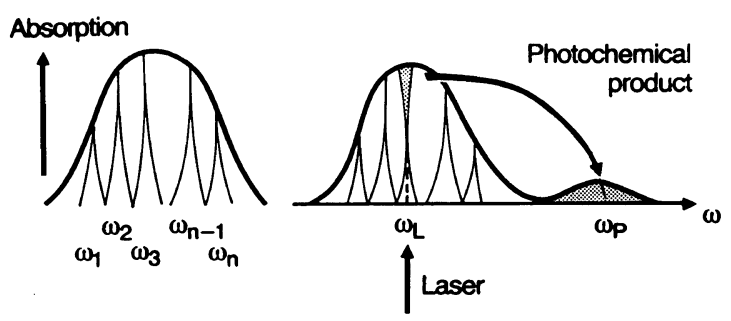

Figure 17. Photochemical hole burning, basic principles. a

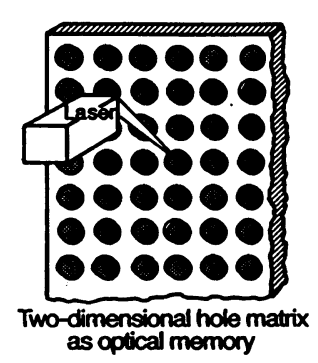

b

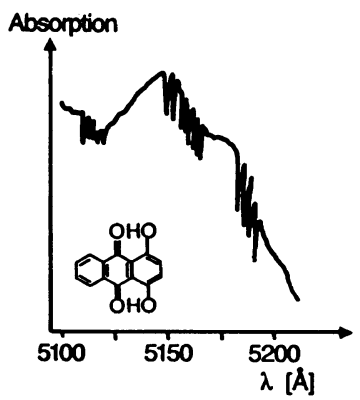

Figure 18. a) Schematic diagram of an optical memory based on photochemical hole burning. b) Absorption band of 1,4-dihydroxyanthraquinone (integrated into an ethanol/methanol glass). 
width of the dyestuff molecule $T_{n}$ to the inhomogenous line width of the dyestuff in the polymer $T_{i}$ lies between $1: 1,000$ and 1 : 10,000 .

If the dyestuff in the polymer is illuminated with frequency-stabilized laser light, the line width which is comparable to or smaller than that of the pure dyestuff exites only those molecules whose absorption band matches the frequency band of the laser light. If a suitable dyestuff is used, the photochemical product of this reaction will not display any absorption within the inhomogenous broadened absorption band. After such excitation the absorption spectrum of the dyestuff-doped polymers displays a hole of the same width as the original (homogenous) dyestuff line at the laser frequency (Figure 17). ${ }^{21}$

This process can be used for storage purposes (Figure 18). ${ }^{21}$ The bit density of the twodimensional memory is limited by the wavelength of the laser light (approx. $10^{8} \mathrm{bit} / \mathrm{cm}^{2}$ ); a packing density of approx. $10^{11} \mathrm{bit} / \mathrm{cm}^{2}$ seems to be possible for $T_{i} / T_{n}=10^{3}$ using frequency-selective read/write processes.

Memories based on photochemical hole burning are thus not pure polymer memories. The bit signals are only differentiated by in- teraction of the polymer matrix and integrated dyestuff molecules.

In addition to the high packing densities, other advantages of memories based on photochemical hole-burning include their multiplex properties, because information can simultaneously be read and written on different frequency channels. This increases the data transmission rates, a factor which is just as important as the packing density in data processing technology.

The following conditions must be satisfied before photochemical hole-burning can be utilized for storage purposes:

-Integration of dyestuff molecules with $T_{i} \gg T_{n}$ into an amorphous polymer.

- No destructive photochemical reaction in the matrix during the reading process. ${ }^{24}$

-Optimum reproduction and maximum frequency stability of the laser light.

Comparison of Magnetic Data Memories and Optical Data Memories. Expected Future Developments

A comparison of the storage material costs per MByte clearly reveals the cost advantages of optical data memories:

\begin{tabular}{|c|c|c|}
\hline Type of memory & Storage capacity & Costs per MByte \\
\hline $\begin{array}{l}\text { Magnetic memories: } \\
\text { - Rigid disk } \\
\text { - Winchester disk } \\
\text { - Floppy disk } \\
\text { - Magnetic tape } \\
\quad(\text { e.g., } 30 \mathrm{~cm} \text { reel approx. } 240 \mathrm{MByte})\end{array}$ & $\begin{array}{c}\text { 1-3 GByte } \\
100 \text { MByte } \\
1 \text { MByte } \\
64 \text { MByte } / \mathrm{km}\end{array}$ & $\begin{array}{l}80 \text { US } \$ \\
8 \text { US } \$ . .80 \text { US } \$ \\
8 \text { US } \$ 16 \text { US } \$ \\
0.8 \text { US } \$\end{array}$ \\
\hline $\begin{array}{l}\text { Optical memory: } \\
- \text { CD-ROM }(\phi 30 \mathrm{~cm})\end{array}$ & 1-3 GByte & $\begin{array}{l}0.8 \text { US } \$ \\
\text { (1990 approx. } 0.08 \text { US \$) }\end{array}$ \\
\hline
\end{tabular}

Figure 19 shows the situation in graph form. ${ }^{24}$ The additional advantages of optical data memories in comparison with magnetic storage media is the 10 to 100 times greater storage density, their insensitivity to dust and fingermarks, as well as the reduced susceptibility of the hardware to failure; it should be remembered in this context that the gap between the magnetic disk and the read/write head in a Winchester drive is only $0.3 \mu \mathrm{m}$. 


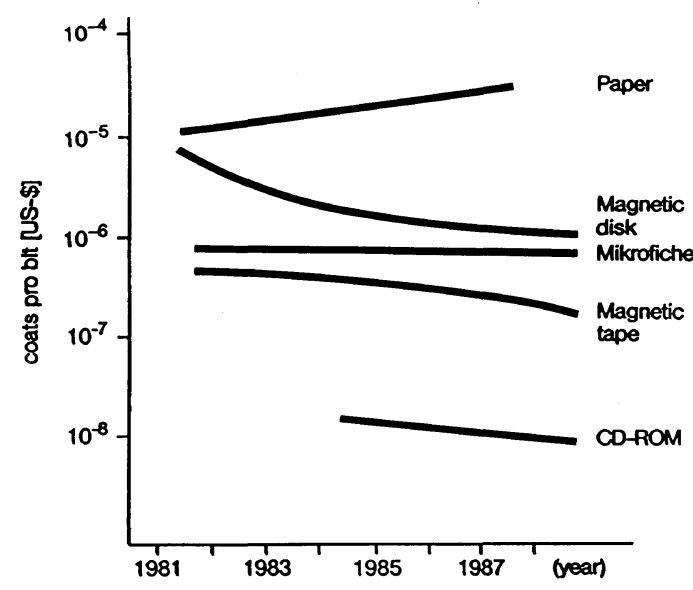

Figure 19. Data storage media: costs per bit (US-\$).



Figure 20. Data storage media of high capacity in western Europe (1984-1993). (Mackintosh study "High Capacity Information Storage.")

The major advantage of magnetic storage media, and one which is difficult to calculate, is the fact that they have established a worldwide market position in what is proving to be a relatively conservative media landscape. In addition, the storage density of magnetic storage techniques is currently increasing at about $30 \%$ per year due to the introduction of improved coating media (rice-grain pigments, barium ferrites, improved Co-doped magnetites, pure iron pigments and thin-film memories) and improved storage methods (e.g., Perpendicular Recording Technique).
In the next two years, the storage density of magnetic media is expected to rise to approx. 2 to $5 \times 10^{7} \mathrm{bit} / \mathrm{cm}^{2}$ (storage costs also declining proportionally). Furthermore, optical memories have almost reached their limit storage density of approx. $6 \times 10^{7} \mathrm{bit} / \mathrm{cm}^{2}$; a further increase would only be possible after the development and introduction of inexpensive diode lasers operating in the visible or near UV range.

It can be foreseen that optical storage media will never achieve the great break-through in replacing magnetic techniques unless success is achieved in the next two years in developing inexpensive, erasable optical data memories with a good signal-to-noise ratio (refer to Section 3). This consideration is also taken into account in the Mackintosh study "High-Capacity Information Storage", which does not forecast a break-through of erasable optical data memories until 1993, at least in Western Europe (Figure 20). ${ }^{22}$

\section{REFERENCES AND NOTES}

1. H. Friedrich, Siemens-Zeitschrift, 59, 19 (1985).

2. G. Kaempf, Ber. Bunsenges. Phys. Chem., 89, 1179 (1985); Techn. Rundschau (Bern), 78, 72 (1986); G. Kaempf, H. Loewer, and M. W. Witman, Polymer, in print.

3. D. J. Gravesteijn and J. van der Veen, Philips Techn. Rev., 41, 325 (1983/84).

4. By courtesy of Philips Research Laboratories, Eindhoven, The Netherlands.

5. H. G. Heller, Br. Patent No. 24.41.759 v. 13.03.75; H. G. Heller, "The Development of Photochromic Compounds for Use in Optical Information Stores," Chem. Ind. London, No. 6 (18.03.1978), p 193; H. G. Heller, IEE Proc., 130, 209 (1983); C. J. G. Kirkby, K. Cush, and I. Bennion, Optics Commun., 56, 288 (1985).

6. S. V. Pappu, Contemp. Phys., 26, 479 (1985).

7. J. Hennig, Kunststoffe, 75, 425 (1985).

8. J. Hennig, paper presented at Bad Nauheim (FRG) on 15.04 .86 , in print.

9. Chemical Daily v. 21.02 .85 .

10. R. Rieß and H. Loewer, Proceedings, SPE 43rd ANTEC, 1985, p 470.

11. J. Furukawa, T. Kotani, and S. Yamashita, J. Appl. Polym. Sci., 13, 2541 (1969).

12. B. R. Hahn and J. H. Wendorff, Polymer, 26, 1619 
(1985).

13. Chem. Eng. News., April 18 (1982).

14. H.-W. Schmidt, doctoral thesis, Mainz (1985).

15. A. I. Lovinger, "Developments in Crystalline Polymers," Vol. I, D. C. Bassett, Ed., Applied Science, London, 1972, Chapter 5; Science, 220, 1115 (1983).

16. C. F. Pulvari, US Patent 2698928 (1955); C. F. Pulvari, IRE Transact., Comp. Parts, 3 (1956); D. S. Campell, J. Br. IRE, 385 (1957).

17. DOS 2216805 vom 08.04.1971.

18. H. Niitsuma and R. Sato, Ferroelectrics, 34, 37 (1980).

19. Prof. Takeo Furukawa, Institute of Physical and Chemical Research, University of Saitama, Japan, private communication.

20. S. E. Cummins and B. H. Hill, Proc. IEEE, 58, 938
(1970); D. Byatt, US Patent 4059827 (1977).

21. G. Castro, D. Haarer, R. M. MacFarlane, and H. P. Trommsdorff, US Patent 4101976 (1978); J. Friedrich and D. Haarer, Angew. Chem., 96, 96 (1984).

22. Mack Intosh-Study, "High Capacity Information Storage."

23. G. Kempf, H. Loewer, and M. Witman, Kunststoffe, 76, 1077 (1986) and German Plastics, 76, 42 (1986); G. Kaempf, Techn. Rundschau (Bern), in print.

24. Chem. Eng. News., April 14, 1986; "New Materials for Storage of Data at High Densities Show Promise."

25. M. Isobe and S. Imai, "Molding Compounds for Optical Disk Substrates: Molecular structural consideration relating for the birefringence of injectionmolded disk." Sumitomo Chem. Co., Ltd., Chimical Research Laboratories (1986). 